\title{
Review and Analysis of Present over the Shoulder Launch Control Technology on Aircraft
}

\author{
Zhangsen $^{1}$,Shanggen Feng ${ }^{1,}$, Pujie $\mathrm{xin}^{1}{ }$ Zhangyuan heng $^{1}$ \\ ${ }^{1}$ College of Information Engineering, Henan University of Science and Technology, Luoyang, \\ 471023, China \\ aemail:sgenfeng_hkd@163.com
}

Keywords: OTSL; Fire control system; Capture zone; Gesture control; Guidance rate

\begin{abstract}
Because of modern air combat is fierce, the air combat situation vary from minute to minute, air combat opportunity transient, and the biggest threat to air combat comes from the tail, so the air combat both are considering how to directly attack the rear target missile, OTSL(over the shoulder launch) is a new way to meet this need and to develop. Study on OTSL plays a key role in the future war. Through the research of the fire control system of missile launching, how to expand the capture zone, implementation of gesture control requirement, guidance rate, the trajectory optimization problem of missile horizontal turning, It enables us to have a further understanding to the OTSL.
\end{abstract}

\section{Introduction}

Air combat plays a decisive role in the future war, so future air battle requires the missile has stronger motor performance, off-axis emission ability, a full range of attack ability, especially for the rear attack, that is the OTSL ability. OTSL has shot after and shot before the two ways: Shot before is the missile forward launch, turning in the air, and then go on to attack rear targets. This kind of method is the aircraft depends on the back target detector, obtain the rear target information, launch with the shoulder capability of missile, turning in the manipulation of loading machine, that is the "over the shoulder", and then to attack the target. The shot after is the missile directly emitted backward, then to attack rear targets. The way is aircraft depends on the back target detector, obtain the rear target information, the missile launching with post shooting ability, the missile fly directly after to attack the rear target, this machine can separate from the carrier[1]. In the first shooting hand, domestic and foreign scholars have done a lot of research, having put forward different compound guidance missile guidance, obtained simulation validation[2-4]. The movement of target and the machine influence each other, this machine can influence the interception of maneuvering target missile, by improving the machine motor, that is to adopt in this machine intelligent maneuver strategy to obtain more capture zone of OTSL. In order to avoid the shortcomings that program control missile turning is not flexible enough, aircraft can not rapidly adjust missile steering according to the target maneuvering, and put forward the method of guiding missile turn and attacking the target. After the missile launching missile turning trajectory also needs to be optimized.

\section{Design of the system}

\section{OTSL fire control system}

OTSL fire-control principle is the machine use airborne radar system and rear-view radar system to probe related information of target, the calculation of fire control area, determine the launch conditions. After the missile launch, the fire control system complete information exchange and command control of among missile, target, the machine. Missile trajectory correction according to the azimuth information of target . After meet the switching conditions, missile into terminal guidance, that is it can automatically attack targets. 


\section{The design of fire control system}

According to the incomplete information of the target, design a sub optimal fire control system. The design idea is firstly according to the characteristics of incomplete information of target fire control based on OTSL, determine the optimal objective function of J control system; Secondly, according to the need to extract the appropriate state variable, composed of nonlinear system; Then by the system model and objective function of nonlinear system to determine the matrix $\mathrm{A}(\mathrm{x})$, $\mathrm{B}(\mathrm{x}), \mathrm{C}(\mathrm{x}), \mathrm{D}(\mathrm{x})$; Finally, according to the structural theory of SDRE to structure locally asymptotically stable suboptimal feedback control system. The initial conditions are as follows: The aircraft and the target in the same flight height $5000 \mathrm{~m}$, apart $7000 \mathrm{~m}$, target azimuth $130^{\circ}$, aircraft and the target fly in $200 \mathrm{~m} / \mathrm{s}$ speed, the implementation of target tracking aircraft, the simulation results such as table $1[5]$ :

\begin{tabular}{|l|c|c|c|c|}
\hline & Missile flight time & Miss distance & Maximum overload & Missile trajectory \\
\hline A & $16.2 \mathrm{~s}$ & $5.53 \mathrm{~m}$ & $30 \mathrm{~g}$ & $\mathrm{a}$ \\
\hline $\mathrm{B}$ & $11.7 \mathrm{~s}$ & $6.20 \mathrm{~m}$ & $49 \mathrm{~g}$ & $\mathrm{~b}$ \\
\hline
\end{tabular}

$\mathrm{A}=$ Correction of non complete information of target azimuth

$\mathrm{B}=$ Sub optimal fire control system

Table 1 The sub optimal fire control system simulation results

\section{Studying on the robustness of the fire control system}

Through the establishment of airborne warning system error model, using of the missile hit probability Monte Carlo simulate method to draw the missile hit probability contour (Figure 1) and the probability of hitting surface (Figure 2) to analyze the effect of measurement error of airborne warning equipment to robustness of the OTSL fire control system. Analysis and founding, at the height of 5000m above, when the airborne detection equipment measure target angle error variance $\sigma=10^{\circ}$, the hitting probability of missile can still guarantee certain in quite a large area, illustrating that has very strong robustness[6]:

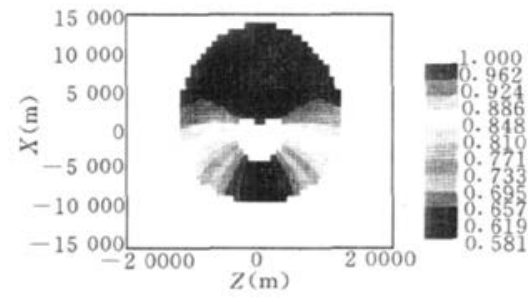

Figure 1 hitting probability contour

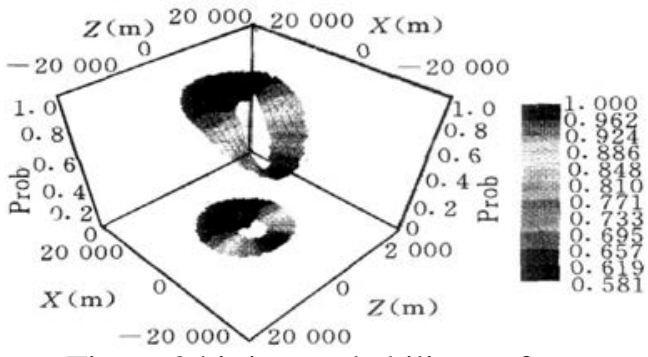

Figure 2 hitting probability surface

Fire control system is an important part of missile launching, researching on fire control system can make the machine without big motor to attack target of all directions, which can realize omnidirectional attack of taking the computer as the core to take the initiative to attack targets.

\section{Research of missile attitude controling}

At present, the OTSL has two direct force control: One is the use of air power and rocket engine or in the relative centroid distance injection pulse micro rocket engine system (torque control) combined with; Two is the use of air power and placing in the missile near the engine (or pulse engine system) (force control) combined with[7].

\section{Direct force / aerodynamic control system}

Direct force control driving mechanism is a discrete actuator with switch properties, therefore, the direct force / aerodynamic driven aircraft control system is a kind of mixed continuous / discrete control system, which puts forward a challenge to the design of the control system. At present for the design problems of direct force / aerodynamic compound control system, solutions are mainly two categories, design method and design method based on joint distribution. There are static allocation and dynamic allocation based on allocation method, static allocation is divided into dynamic pressure distribution method, chain distribution method, linear programming method. Joint 
design method is to take the aerodynamic control loop as the inner loop, based on continuous control theory to design controller, and then take the aerodynamic control system as the controlled object, design the control system of direct force[8].

\section{Static allocation}

$\mathrm{M}_{\mathrm{c}}$ is the general control torque command that controller calculate according to tracking command and sensor feedback information. The commonly used dynamic pressure distribution algorithm is:

$$
\begin{aligned}
& M_{\text {thrust }}=f(q) M_{c}, M_{\text {aero }}=(1-f(q)) M_{c} \\
& f(q)=\frac{q_{2}-q}{q_{2}-q_{1}}
\end{aligned}
$$

In the formula: $\mathrm{q}_{1}$ and $\mathrm{q}_{2}$ is the ideal state of dynamic pressure size before and after the phase transition, $\mathrm{q}$ is the current dynamic pressure.

Chain distribution method refers to the preferred selection of pneumatic rudder control alone, after the pneumatic rudder reached full partial, the direct force subsystem start to take part in the control.

Using linear programming control allocation is intended to find the saturation bounded pneumatic rudder control quantity and the engine ignition number (number) of the optimal portfolio, so the precision is satisfied flight control, making control energy consume minimum.

The combined design method is to combine the static allocation with dynamic allocation method, the main design idea is to design the aerodynamic force feedback control loop, and then take the pneumatic control system as the controlled object, and then the design of direct force feedback control system.

\section{Pulse jet control system}

As shown in Figure 3 is a body layout, there are 180 pulse engine be arranged in front of the center of mass. Pulse engine uses the adjacent ring staggered, not each circle and each angle around the missile has the attitude engine, but every fixed point of view have the impulse engine.

Make from centroid the nearest first lap engine to missile centroid distance as $L_{T 1}$, the distance of adjacent ring is $\lambda$, the $j(j=1,2, \cdots 10)$ ring engine to centroid distance is $L_{T j}$, dividing into 36 columns, each column has 5 pulse engine, namely $i=1,2 \cdots, 36$.so:

$$
L_{T j}=L_{T 1}+(j-1) \lambda \quad(j=1,2, \cdots, 10)
$$

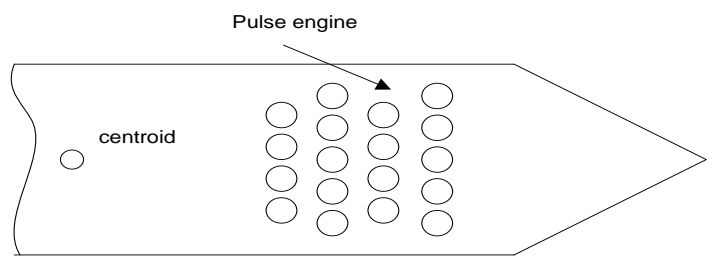

Figure 3 Missile head shape is shown in Fig

When the pulse engine works, the pulse force to the centroid distance is $L_{T}$, if the working engine is located in the $\mathrm{j}$ ring, so $L_{T}=L_{T j}$, a single pulse engine thrust $T$ is $T=K_{f} N, N$ is pulse net thrust engine, $K_{f}$ is pulse engine thrust factor.

Simulating in missile terminal passive, in the case whose distance to target the initial axial distance $\mathrm{X}$ is about $1000 \mathrm{~m}, \mathrm{Y}$ axial is about the $7000 \mathrm{~m}$ to pursue the target. The initial target speed is $750 \mathrm{~m} / \mathrm{s}$, the initial load is zero. The results of adding straight relay and without direct force is shown below[9]. 


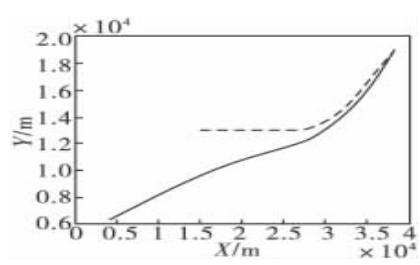

Figure 4 Having direct force

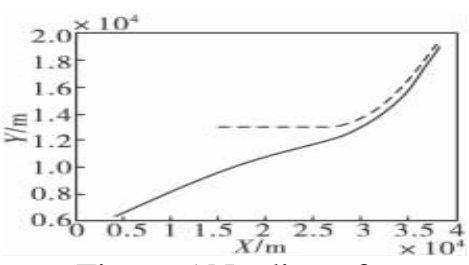

Figure 5 No direct force

The dotted line represents the trajectory of moving object, the solid line shows the trajectory of missile, when no having direct force, the miss distance is $352.4 \mathrm{~m}$, having direct force the miss distance is $0.495 \mathrm{~m}$. The results show that the accurate against target pulse engine added can effectively control the attitude of the missile.

The design of the controller has sliding mode variable structure control, variable structure control, pitch yaw channel control etc.

\section{Missile guidance}

Currently, the research on the guidance rate has based on guidance of beam guidance, based on guidance of large off axis, based on the attack guidance rate of direct force / aerodynamic compound control, based on variable structure guidance rate of data chain.

\section{Based on guidance of beam guidance}

Beam guided missile attack rear target process is: Aircraft utilization to target detector to obtain the target information (range, azimuth), after the conditions allowing missile launch, then carrier transmitting beam to guide missile. Beam with a certain angular velocity $\omega_{t}$ toward the back of machine rotation, the size and direction of rotation of $\omega_{t}$ can be adjusted[10]. After reaching to the maximum radar deflection angle, by the wireless transmitting unit on both sides of the fuselage transmitting electromagnetic waves continue to guide the missile. In order to omni-directional guided missile, can fix rolling spherical pod for load machine to transmit beam or mounted the side guide antenna in fuselage two ends[11]. After the missile intercept target, beam end, the missile fly to the target by proportional navigation method. As shown in Figure 6[10].Beam guided missile simulation schematic diagram as shown in Figure 7[10]:

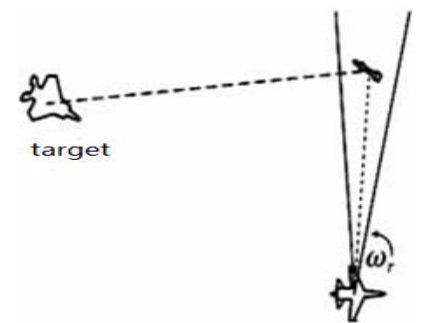

Figure 6 Missile turn map

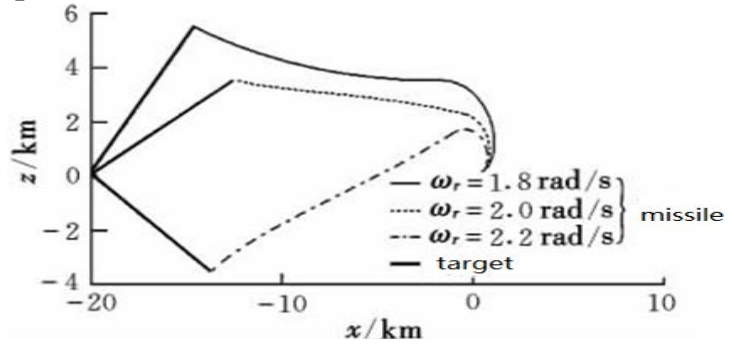

Figure 7 The relationship of ballistic and $\omega_{t}$

\section{Based on direct force / aerodynamic compound control attack guidance}

The guidance consists of two parts: One part is the over the shoulder turn period guidance, other is the guidance rate that missile have completed over the shoulder.

turn period guidance

Through the procedures for fast turning trajectory optimization design to realize the missile fast turning. Considering the over the shoulder turning in the horizontal plane, steering force is mainly composed by the sideslip angle and the body $\mathrm{Z}$ overload to produce, y overload mainly keep Missile Altitude basically unchanged. $\mathrm{Z}$ overload and y overload is:

$$
\begin{aligned}
& n_{y}=K(V \dot{\theta}+g \cos \theta) \\
& n_{z}=K\left(-V \cos \theta \dot{\psi}_{c}\right)
\end{aligned}
$$

K gets 1.2-1.5.

The guidance rate that missile have completed over the shoulder 
Using the extended proportional guidance law based on the target acceleration compensation, get the control instruction of line of sight coordinate system based on target acceleration compensation, the expansion ratio guidance rate is:

$$
\left\{\begin{array}{l}
A_{y b}=\frac{A_{y s}}{\cos \alpha}-A_{z s} \tan \alpha \tan \beta+A_{x b} \frac{\tan \alpha}{\tan \beta} \\
A_{z b}=\frac{A_{z s}}{\cos \beta}-A_{x b} \tan \beta
\end{array}\right.
$$

A is missile control system command, $\beta$ is azimuth, $\alpha$ is angular altitude.

The typical simulation conditions as in Table 2:

\begin{tabular}{|c|c|c|c|c|c|c|c|c|}
\hline Order number & $H$ & $V_{M}$ & $V_{T}$ & $\vartheta$ & $\theta$ & $\lambda$ & $D$ & $A_{C}$ \\
\hline 1 & 5 & 0.8 & 0.8 & 140 & 0 & -10 & 5 & 6 \\
\hline 2 & 5 & 0.8 & 0.8 & 180 & -20 & 0 & 8 & 0 \\
\hline 3 & 10 & 1.0 & 0.8 & 120 & 0 & 20 & 8 & 3 \\
\hline 4 & 10 & 1.0 & 0.8 & 150 & 20 & 60 & 10 & 0 \\
\hline 5 & 15 & 1.2 & 0.8 & 90 & -10 & 180 & 10 & 0 \\
\hline 6 & 15 & 1.2 & 1.2 & 120 & 0 & 30 & 10 & 0 \\
\hline
\end{tabular}

Table 2 The typical simulation conditions

$\mathrm{H}$ is missile altitude, $\mathrm{km}, V_{M}, V_{T}$ is the missile and the target emission speed Ma, $\vartheta, \theta, \lambda$ are relative target missile azimuth angle, elevation angle and velocity direction angle, $\left({ }^{\circ}\right), \mathrm{D}$ is the initial firing distance, $\mathrm{km}$; Ac is the target maneuver magnitude.

The results show the feasibility of this guidance rate.

Simulation results is shown in Figure 8,9[12].
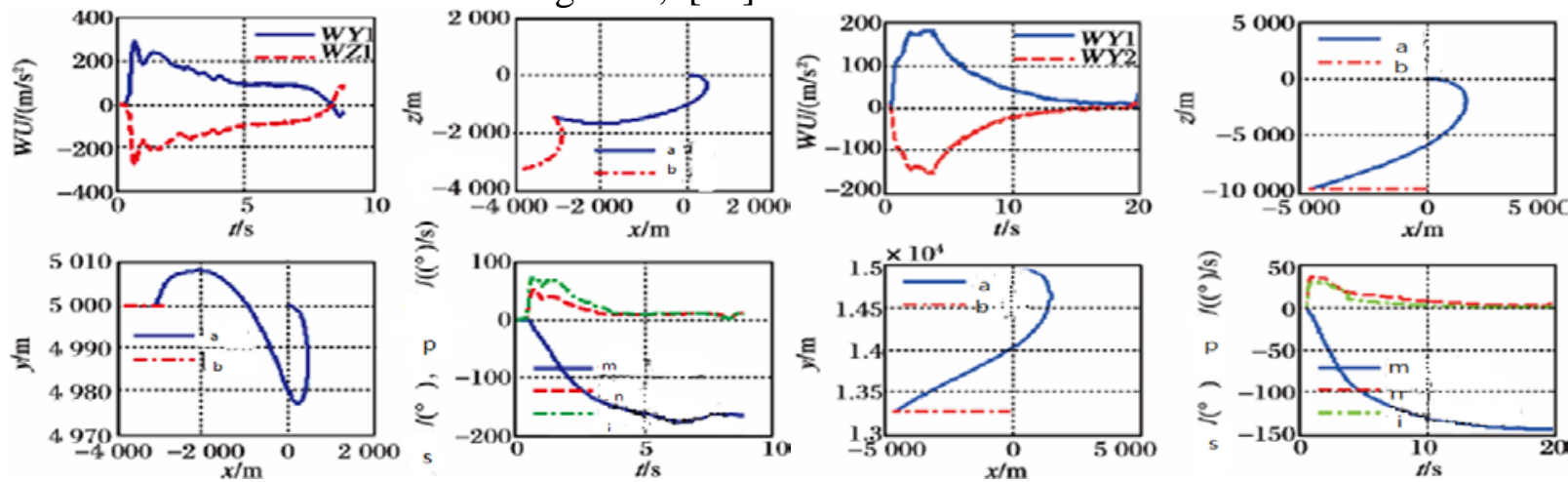

Figure 8 The simulation 1
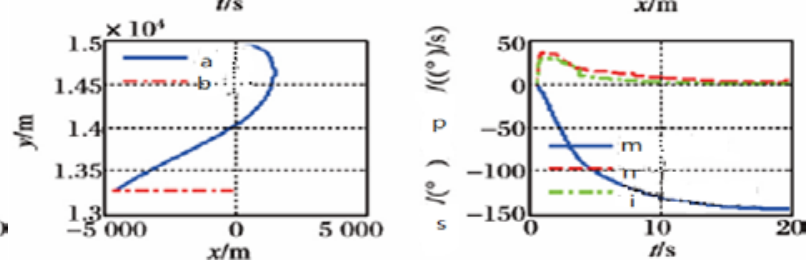

Figure 9 The simulation 5

$\mathrm{a}=$ missile; $\mathrm{b}=$ target; $\mathrm{p}=$ palstance;s=angle;m=velocity deflection; $\mathrm{n}=$ angle of attack;i=the turing palstance

\section{Based on variable structure guidance rate of data chain}

Interceptor missile generally strike high speed running of the object, the goal is constantly changing, so mounting on the data link system in the missile. When in work, in the middle guidance flight, by the movement of airborne radar measurement information of the target, passing the target motion parameters to the missile through the data chain system, by the missile strapdown inertial navigation system and apparatus for measuring a target motion parameters, through the onboard computer to form the control instruction according to the guidance law is established, controling missile stabilitly fly to the target according to the scheduled ballistic[13].

The equation of motion of missile: 
$\left\{\begin{array}{l}\dot{x}_{M}=v_{M} \cos \sigma_{M}, x_{M}(0)=x_{M 0} \\ \dot{y}_{M}=v_{M} \sin \sigma_{M}, y_{M}(0)=y_{M 0} \\ v_{M}=a_{M h}, v_{M}(0)=v_{M 0} \\ \dot{\sigma}_{M}=a_{M v} / v_{M}\end{array}\right.$

Target motion equation:

$\left\{\begin{array}{l}\dot{x}_{T}=v_{T} \cos \sigma_{T}, x_{T}(0)=x_{T 0} \\ \dot{y}_{T}=v_{T} \sin \sigma_{T}, y_{T}(0)=y_{T 0} \\ v_{T}=a_{T h}, v_{M}(0)=v_{T 0} \\ \dot{\sigma}_{T}=a_{T v} / v_{T}\end{array}\right.$

$x_{M 0}, y_{M 0}, v_{M 0}, x_{T 0}, y_{T 0}, v_{T 0}$ are the initial conditions of missile and target moving, $a_{M h}$, $a_{M v}, \quad a_{T h}, a_{T v}$ is the tangential and normal acceleration of missile and the target.

This scheme is derived for guidance rate:

$\dot{a}_{M v}=\left(C-B a_{M v}\right) / A$

Inside:

$$
\begin{aligned}
& A=r / v_{M} ; B=c+\dot{r} / v_{M}+\cos \left(\sigma_{M}-q\right)-r a_{M h} / v^{2}{ }_{M} \\
& C=\left(c v_{M}-\dot{r}\right) q+f-a_{M h} \sin \left(\sigma_{M}-q\right)+c a_{M h}\left(q-\sigma_{M}\right)+(k|\dot{r}| / r) s+\varepsilon \operatorname{sgn}(s)
\end{aligned}
$$

Type parameters refer to the literature 14

The simulation is to assume that the target yaw movement, speed: $300 \mathrm{~m} / \mathrm{s}$, the maximum motor overload is $10 \mathrm{~g}$, when the distance is $50 \mathrm{~km}$ to aircraft, beginning snaking motion, initial position is $(60000 \mathrm{~m}, 10000 \mathrm{~m})$, missile velocity, $600 \mathrm{~m} / \mathrm{s}$, the maximum motor overload is $20 \mathrm{~g}$, initial position is $(0 \mathrm{~m}, 0 \mathrm{~m})$, the locking range of radar seeker is $18 \mathrm{~km}$, assuming hypothesis guidance section the initial deviation of missile velocity direction is $-3^{\circ}$. The simulation results as shown in Figure 10-12[13].

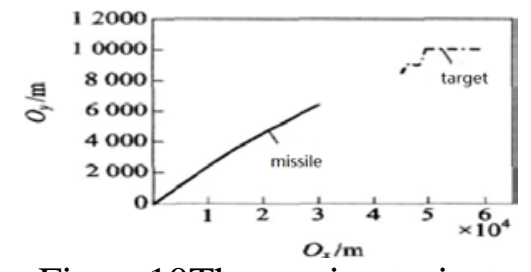

Figure10The motion trajectory

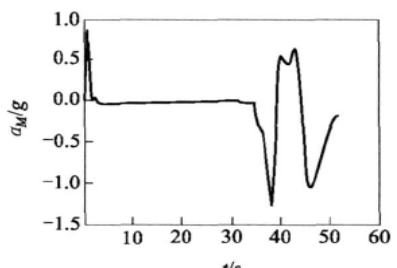

Figure 11 the overload chaning

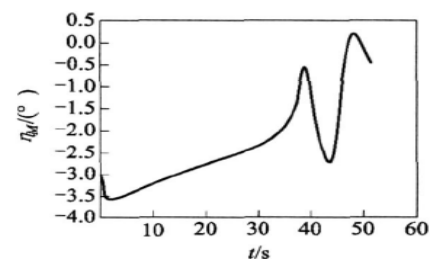

Figure 12 heading angle

From the simulation results, see guidance handover missile velocity heading angle and normal overload are very small, excellent performance, can meet the control requirements of the missile.

OTSL is a new battle mode, can improve the missile's combat capability, the research of guidance is the key to combat mode. In the current technology, guidance in the form of data chain control will be of great used space. Data link control system can more easily provide varying time enemy, constantly adjust to the missile, attack targets accurately.

\section{Conclusion}

As a new airborne fire control technology, the OTSL is different with the past attacking methods, through the deep study of OTSL, and proposes a new idea and new problems with universality to airborne fire control technology. This is a new challenge for airborne fire control research, more is a development opportunity. Aviation fire control technology from forms of the research content and theory to the theoretical model and hypothesis of the face is really a revolution, OTSL will become 
an important way in future war.

\section{Acknowledgement}

In this paper, the research was sponsored by the Aviation Science Fund (Project No. 20130142004)and the National Natural Science Fund((Project No.61304144).

\section{References}

[1] He Su Juan. Air to air missile OTSL guidance research[D]. Northwestern Polytechnical University. 2006.3

[2] Taur D R, Chern J S. Optimal Thrust Vector Control of Tactical Missiles[C]//Proceedings of the AIAA Guidance, Navigation, and Control Conference. 1997. (AIAA-97-3475)

[3] Taur D R, Chern J S. An Optimal Composite Guidance Strategy For Dogfight Air-To-Air IR Missiles[C]// Proceedings of the AIAA Guidance, Navigation, and Control Conference. 1999. (AIAA-99-4066).

[4] Cloutier J R, Stansbery D T. All-Aspect Acceleration-Limited Homing Guidance[C]// Proceedings of the AIAA Guidance, Navigation, and Control Conference. 1999. (AIAA-99-4063).

[5] Cai Fu Guang, Gao Xiao Guang. The research of OTSL sub optimal fire control system[J]. Fire control and command.2002.8

[6] Li Bo. The robust research of OTS fire control system[J]. Fire control and command.2007.7

[7] Chen Wei Min, Huang Shao Bo, He Yong Jie. A preliminary study on the direct force control device of air to air missile[J]. Aviation Weapon. 2009.2

[8] Liang Dong, Song Jian Mei, Cai Gao Hua. High speed aircraft direct force / Aerodynamic Compound Control Technology Overview[J]. Aviation Weapon. 2013.4

[9] Zhan Jian Chao, Ren Yong Qiang, Zhang Li, Zhu Zhu. The design attitude control pulse engine ignition logic[J]. Journal of missiles and guidance. 2011.12

[10] Li Hao Liang, Zhang Bin, Xu An, Zuo Xing Xing. Based on the study of beam guided air-to-air missile OTS guidance law[J]. Flight Mechanics. 2013.2

[11]Ian Moir, Allan Seabridge. Military avionices systems[M] .England:American John Wiley \& Sons Ltd, 2006:256-65

[12] Zhang Peng, Zhang Jin Peng. Air to air missile OTS guidance research[J]. Aviation Weapon. 2014.3

[13] Qing fang. Missile flight mechanics[M]. Beijing Institute of Technology press.2000.7

[14] Wang Yu Lin, Zhou Shao Lei, Han Zhi Bin. Based on the research on variable structure guidance law intercept missile of data link[M]. Tactical Missile Technology. 2009.11 